https://doi.org/10.22319/rmcp.v11i2.5349

Revisión bibliográfica

\title{
Bolos intrarruminales con liberación controlada de minerales traza. Revisión
}

Misael León-Cruz ${ }^{\mathrm{a}}$

Efrén Ramírez-Bribiesca ${ }^{\mathrm{a}^{*}}$

Raquel López-Arellano ${ }^{\mathrm{b}}$

Leonor Miranda-Jiménez $^{\text {a }}$

Gabriela Rodríguez-Patiño ${ }^{b}$

Víctor M. Díaz-Sánchez ${ }^{\text {b }}$

Alma L. Revilla-Vázquez b

${ }^{a}$ Colegio de Postgraduados. Programa de Ganadería. Campus Montecillo. Km. 36.5 Carr. México-Texcoco. 56230, Estado de México. México.

b Universidad Nacional Autónoma de México, FES Cuautitlán, Cuautitlán Izcalli, Estado de México.

* Autor de correspondencia: efrenrb@colpos.mx

\section{Resumen:}

Los minerales traza son nutrientes esenciales para el mantenimiento de la vida, el crecimiento y la reproducción. Las deficiencias de minerales en rumiantes afectan las funciones fisiológicas y metabólicas que con frecuencia causan enfermedades. El diseño y uso de los bolos intrarruminales de liberación controlada (BILC) es una alternativa para corregir la falta de los microelementos en el organismo. El propósito de esta revisión es evidenciar la información disponible sobre los diferentes tipos de BILC de minerales traza, así como de los métodos de fabricación que incluye: técnicas de extrusión en caliente, 
granulación por fusión y fusión directa. Además, se describen los efectos de BILC relacionados en la salud, en los parámetros productivos y reproductivos en rumiantes.

Palabras clave: Bolos intrarruminales, Mecanismos de liberación, Concentración mineral, Cinética de liberación.

Recibido: 24/04/2019

Aceptado: 21/05/2019

\section{Introducción}

La deficiencia de minerales traza en los pastos y forrajes de diversas regiones ganaderas son comunes en el mundo y en México. Las principales causas son la baja concentración mineral en los suelos y el antagonismo entre minerales que dificulta su disponibilidad para las plantas y animales ${ }^{(1)}$. Los minerales traza como el cobre $(\mathrm{Cu})$, zinc $(\mathrm{Zn})$, cobalto $(\mathrm{Co})$, selenio (Se) y yodo (I) son deficientes en rumiantes ${ }^{(2-5)}$ y ocasionan trastornos en los parámetros productivos y reproductivos ${ }^{(1)}$.

Ante esta problemática la industria alimentaria y farmacéutica veterinaria han desarrollado productos con suplementos de minerales traza en forma de: premezclas minerales, bloques $\mathrm{y}$ soluciones inyectables, pero se requieren dosificaciones frecuentes, y el consumo es variable cuando se suministran a libre acceso ${ }^{(6,7)}$. Los bolos intrarruminales de liberación prolongada (BILC) son dispositivos diseñados para ser administrados por vía oral y deben permanecer en el retículo-rumen por periodos de tres meses o hasta un año ${ }^{(8,9)}$, considerado el método más indicado para corregir deficiencias de los minerales traza en rumiantes en pastoreo $^{(6,10,11)}$.

El diseño de BILC se puede realizar con los siguientes criterios básicos: las dimensiones, la geometría de los sistemas de liberación y la densidad. Estos criterios determinan la forma de liberación de los minerales; para ello se han utilizado materiales (excipientes) que mediante diversos mecanismos controlan la velocidad de dosificación ${ }^{(8,9)}$. En esta revisión se describen los diferentes tipos de BILC de minerales traza, así como los métodos de fabricación, considerando la extrusión en caliente, granulación por fusión y fusión directa. Además, se discuten los efectos de BILC en la salud, en los parámetros productivos y reproductivos de rumiantes. 


\section{Bolos intrarruminales de liberación controlada}

Los BILC son dispositivos sólidos que pueden liberar minerales traza, fármacos, promotores de crecimiento y nutrientes en el retículo-rumen de los rumiantes ${ }^{(8,9)}$. Deben cumplir con ciertas características para permanecer en el retículo-rumen y liberar la dosis correcta, de manera inmediata o controlada. En el diseño se consideran tres criterios básicos $^{(8,9,12,13)}$ :

Dimensiones. El diámetro de los bolos debe ser alrededor de $25 \mathrm{~mm}$ con longitud variable de 40 a $100 \mathrm{~mm}$.

Geometría de los sistemas de liberación. Comúnmente la forma es cilíndrica o esférica, con puntas redondas y superficie lisa o con forma de cápsula, de manera que se pueda administrar por vía oral. Sin embargo, existen diseños con alas plegables, hojas y anillos compresibles que se convierten en forma expandida, para evitar la regurgitación y permanecer en el retículo-rumen durante el tratamiento.

Densidad. Debe ser superior a $2.0 \mathrm{~g} \mathrm{~cm}^{-3}$ para garantizar la permanencia del bolo en el retículo-rumen. Esta puede lograrse al incluir en la formulación un agente densificador como el hierro $(\mathrm{Fe})$ o vidrio soluble.

\section{Bolos comprimidos}

Este tipo de bolo está compuestos por una matriz comprimida que contiene fármacos o minerales traza, y pueden estar recubiertos por membranas poliméricas ${ }^{(14)}$. Se diseñaron para disolverse o desgastarse por acción mecánica del rumen ${ }^{(8)}$, por lo tanto, la liberación del fármaco o minerales traza ocurre mediante la erosión o difusión del bolo durante periodos predeterminados ${ }^{(14)}$. Se determinó que densidades superiores a $2.0 \mathrm{~g} \mathrm{~cm}^{-3}$ es suficiente para asegurar la retención del bolo en el retículo-rumen y evitar la regurgitación ${ }^{(15)}$. Por ejemplo en Australia desarrollaron un bolo erosionable a base de óxido cobáltico $\left(\mathrm{Co}_{3} \mathrm{O}_{4}\right)$ y otros diluyentes para suministrar Co a rumiantes en pastoreo ${ }^{(16)}$. Los bolos contenían entre 30 a $90 \%$ de $\mathrm{Co}_{3} \mathrm{O}_{4}$, con peso variable de 5.5 a $30 \mathrm{~g}$, y resultaron eficaces para proporcionar Co hasta por más de un año ${ }^{(16,17)}$. A pesar que los bolos tenían un peso específico entre 3.5 y $4.1^{(17)}$, presentaron problemas de regurgitación, así como de formación de una capa de fosfato de calcio en la superficie, que impidió la correcta disolución del bolo ${ }^{(7,17)}$. Para disminuir la incrustación de fosfato de calcio en el bolo se optó por administrar un tornillo de acero que al hacer contacto con el bolo las abrasiones desintegran los fosfatos de calcio ${ }^{(17,18)}$. Este problema también se observó en bolos de pellets de Se formulados con 5 o $10 \%$ de Se y 90 o $95 \%$ de Fe, los cuales tenían un peso aproximado de $10 \mathrm{~g}$, con $12 \mathrm{~mm}$ de diámetro y $15 \mathrm{~mm}$ de largo ${ }^{(19)}$. Sin embargo, algunas 
formulaciones resultaron poco efectivas, aseverando que el tamaño de la partícula de Se utilizada en la fabricación de los pellets fue el factor limitante en la liberación de Se a largo plazo $^{(18,20)}$. De manera similar desarrollaron bolos de pellets de $\mathrm{Zn}$ a base de la mezcla de 5 g de Zn y 5 g de limadura de Fe. Los bolos presentaron tasas de liberación de 10 mg de Zn día ${ }^{-1(21)}$.

Varios autores han desarrollado bolos comprimidos diseñados para suministrar Se a $\operatorname{caprinos}^{(22)}$ y ovinos ${ }^{(23,24)}$. Por ejemplo, en un estudio demostraron la efectividad de bolos de 10 g para corregir la deficiencia de Se en ovejas. El bolo se fabricó con $5.23 \%$ de selenito de sodio, $68.77 \%$ de Fe, $25 \%$ de cutina y $1 \%$ de estearato de magnesio ${ }^{(24)}$. Sin embargo, con esta estrategia de suplementación se presentaron problemas de regurgitación, por ello en el diseño se debe considerar la forma geométrica y la densidad del bolo. Para lo anterior, se diseñó un bolo que posee alas poliméricas y se expanden al entrar en contacto con el fluido ruminal, esto evita la regurgitación del bolo. El dispositivo contiene en su interior un resorte que actúa sobre un pistón y que a su vez ejerce presión sobre la matriz erosionable que puede contener fármacos o minerales traza. La liberación se da cuando la matriz entra en contacto con el medio exterior a través de un portal en uno de los extremos del bolo ${ }^{(8)}$.

Con un enfoque diferente ${ }^{(23)}$, se diseñó un bolo para el tratamiento de la coccidiosis en corderos. El bolo consistió en una matriz que contiene $30 \%$ de sulfametazina sódica, $54.4 \%$ de $\mathrm{Fe}$ reducido, $15 \%$ de aceite de ricino hidrogenado y $0.5 \%$ de estearato de magnesio. El peso promedio del bolo fue de $18.5 \mathrm{~g}$ y la densidad de $2.3 \mathrm{~g} \mathrm{~cm}^{-3}$. La liberación de la sulfametazina se produce a través de difusión y erosión de la matriz. Además determinaron que dosis de $800 \mathrm{mg} \mathrm{kg}^{-1} \mathrm{PV}$ y la fuerza de compresión superior a $2,160 \mathrm{~kg} \mathrm{~cm}^{-2}$ es suficiente para mantener liberaciones sostenidas y concentraciones plasmáticas de sulfametazina $\left(>25 \mu \mathrm{g} \mathrm{ml}^{-1}\right.$ ) hasta por $100 \mathrm{~h}$, siempre que la resistencia mecánica de los bolos sea de $33.5 \pm 1.2 \mathrm{~kg}^{(23)}$. Los bolos formulados con selenito de sodio y sulfametazina tienen peso promedio de $20.13 \mathrm{~g}$, densidad de $2.0 \mathrm{~g} \mathrm{~cm}^{-3}$, longitud de $52.05 \mathrm{y}$ $21.22 \mathrm{~mm}$ de ancho. Estos bolos resultaron eficaces para controlar la coccidiosis y mantener el contenido adecuado de sulfametazina y selenio en cabritos ${ }^{(22)}$.

En la fabricación de los bolos se han utilizado diferentes polímeros insolubles en fluidos ruminales, como revestimiento de matrices que contienen fármacos o minerales traza. Los polímeros forman una membrana quebradiza que permite la erosión de la matriz y la liberación de fármacos o minerales traza ${ }^{(25)}$. Un ejemplo de este enfoque es el bolo AllTrace $^{\circledR}$ compuesto de una mezcla comprimida de 30 g que contiene sales inorgánicas (óxido de cobre, selenito de sodio, sulfato de cobalto, yoduro de potasio, sulfato de manganeso, óxido de zinc ( $\mathrm{ZnO})$, sulfato de zinc) y vitaminas $\mathrm{A}, \mathrm{D}_{3}$ y E. En un extremo tiene un contrapeso de $18 \mathrm{~g}$ que asegura la permanencia del bolo en el retículo-rumen, al final de su vida útil se disuelve totalmente sin dejar residuos. El bolo es recubierto por una 
resina de polímero inerte que controla la liberación durante un periodo aproximado de 240 días, excepto el extremo superior que va a estar en contacto con el ambiente ruminal ${ }^{(26,27)}$.

\section{Bolos extruidos}

Este tipo de bolo se obtiene por extrusión de la formulación de minerales traza y excipientes poliméricos ${ }^{(14)}$, el proceso se describe en la sección de métodos de fabricación de bolos. Este bolo fue desarrollado en Nueva Zelanda para el tratamiento del eccema facial. El bolo consiste en una matriz extruida de $\mathrm{ZnO}$ cubierta con un material ceroso e impermeable al fluido ruminal, excepto un extremo que al entrar en contacto con el líquido ruminal se erosiona para liberar el microelemento. A medida en que la matriz se erosiona, el recubrimiento ceroso se desintegra; esto garantiza la exposición constante de una parte de la matriz al medio ruminal ${ }^{(14,28,29)}$.

\section{Bolos de vidrio soluble}

Los bolos de vidrio soluble (BVS) fueron diseñados para suministrar $\mathrm{Cu}, \mathrm{Zn}, \mathrm{Co}$, Se y I a rumiantes en pastoreo ${ }^{(6,11,30-35)}$. La composición consta de pentaoxido de fosforo $\left(\mathrm{P}_{2} \mathrm{O}_{5}\right)$, óxido de sodio $\left(\mathrm{Na}_{2} \mathrm{O}\right)$ y óxido de calcio $(\mathrm{CaO})$, como óxidos formadores y modificadores del vidrio. Esta composición permite una tasa de liberación en el retículo-rumen de no más de $25 \mathrm{mg} \mathrm{cm}^{-2}$ día $^{-1(36)}$, lo que hace posible que puedan administrarse a los rumiantes para liberar minerales traza hasta por más de un año ${ }^{(30,31)}$. La liberación de los microelementos ocurre por difusión o disolución del vidrio, y en mayor proporción por erosión ${ }^{(8,13,30,36,37)}$.

Los bolos para ovejas tienen peso variable de 30 a $35 \mathrm{~g}$, una longitud de 40 a $50 \mathrm{~mm}$, un diámetro de 14 a $19 \mathrm{~mm}$ y densidades de 2.7 a $4.0 \mathrm{~g} \mathrm{~cm}^{-3}$, mientras que los bolos para bovinos son de mayor dimensión; pesos de 100 g, diámetro de 24 a 26 mm y longitud de 80 $\mathrm{mm}^{(36)}$. Otros aspectos que se deben considerar en el diseño de los bolos son el tamaño de la partícula y el $\mathrm{pH}$ del medio de alojamiento ${ }^{(37)}$;esto con el propósito de lograr la tasa de liberación antes citada. Los BVS presentan múltiples ventajas, entre ellas incluyen una menor formación de fosfato de calcio en el bolo ${ }^{(1)}$, lo que facilita la disolución, mejores perfiles plasmáticos ${ }^{(30,31,35)}$, mayores índices productivos y reproductivos ${ }^{(32,35,38,39)}$, así como beneficios económicos ${ }^{(6,11)}$. Sin embargo, durante el proceso de fabricación de BVS no es posible incluir materiales (excipientes o fármacos) sensibles o inestables a las altas temperaturas de formación del vidrio. No obstante, la incorporación de fármacos u otros componentes en el vidrio puede ser mediante procesos de sinterización mediada por presión $^{(37)}$. 


\section{Bolos de magnesio}

Los bolos de magnesio ( $\mathrm{Mg}$ ) consisten en un cilindro formados por aleación de $\mathrm{Mg}$, aluminio y $\mathrm{Cu}(86,12$ y $2 \%$, respectivamente), contienen Fe disperso en la matriz del bolo para aumentar la densidad. Los bolos para ovejas pesan alrededor de $35 \mathrm{~g}$ y se erosionan por influencias electroquímicas en el rumen, liberando el $\mathrm{Mg}$ en un periodo aproximado de tres semanas ${ }^{(40)}$. Otro ejemplo de bolo de $\mathrm{Mg}$ consiste en dos mitades cilíndricas ligadas con una goma para facilitar su administración, cuando el bolo llega a rumen, se abre para evitar la regurgitación y libera por acción electrolítica una tasa aproximada de $2 \mathrm{~g} \mathrm{Mg}$ por dispositivo durante un periodo de tres meses ${ }^{(41)}$.

\section{Cápsulas con alambre de óxido de cobre}

Los alambres de óxido de cobre $(\mathrm{CuO})$ se han utilizado para corregir deficiencias de $\mathrm{Cu}$ en ovinos $^{(21,42,43)}$ y bovinos ${ }^{(44)}$. Miden entre 3 y $12 \mathrm{~mm}$ de largo, 0.5 a $1 \mathrm{~mm}$ de diámetro y tienen gravedad especifica de 6.1 a $6.4^{(42,45,46)}$, generalmente son recubiertos por una mezcla de óxido cúprico y cuproso $^{(21)}$ o contenidos en cápsulas de gelatina ${ }^{(42,46)}$. Los alambres de $\mathrm{CuO}$ se depositan en el retículo-rumen y luego fluyen al abomaso, en donde el ácido clorhídrico los disuelve para liberar iones de $\mathrm{Cu}$ que se absorben en procesos bioquímicos normales ${ }^{(41,46)}$. La administración de cápsulas de alambres de $\mathrm{CuO}$ aumentan la concentración de $\mathrm{Cu}$ hepático durante 6 a 12 meses; esto se debe a la inercia relativa de las partículas en el retículo-rumen y a la retención en el abomaso ${ }^{(21)}$.

\section{Métodos de fabricación de BILC}

\section{Extrusión en caliente}

Esta técnica se emplea para elaborar productos farmacéuticos como tabletas, cápsulas, películas e implantes para la administración de fármacos por vía oral, transdérmica y transmucosa ${ }^{(47,48)}$. El proceso consiste en el bombeo de materias primas a través de un tornillo que gira a temperaturas de 30 hasta $250{ }^{\circ} \mathrm{C}$ dentro de un troquel; consiguiendo una mezcla homogénea de los compuestos activos y aglutinantes (termoplásticos y polímeros). El equipo utilizado comúnmente se denomina extrusora, consta de un barril que contiene bandas que calientan, reblandecen la mezcla de compuestos químicos y la comprime. Finalmente el extruido es conducido hacia la matriz para darle la forma y las dimensiones requeridas ${ }^{(47,49)}$. 


\section{Granulación por fusión}

La granulación por fusión es una técnica basada en la utilización de agentes aglutinantes sólidos, que se funden a temperaturas entre 50 a $80{ }^{\circ} \mathrm{C}$. Esto permite emplearse para la formulación de fármacos o minerales traza sensibles a la humedad, evitando el uso de solventes acuosos u orgánicos ${ }^{(50)}$. El proceso de granulación puede consistir de un solo paso; para lo anterior el agente aglutinante se añade junto con el resto de los componentes de la mezcla a un mezclador granulador de alta velocidad. La fusión del agente aglutinante se da por el calor generado con la corriente de aire durante la fase de mezclado, amasado y secado. Finalmente el granulado se obtiene de la unión entre el aglutinante fundido y las partículas de polvo, una vez seco debe tamizarse con el fin de obtener el tamaño de gránulo deseado $^{(51)}$. Dependiendo de la finalidad farmacéutica, el granulado se puede encapsular para la liberación inmediata del fármaco o comprimir para formar bolos intrarruminales de liberación controlada.

\section{Fusión directa}

Esta técnica consiste en fundir la mezcla de componentes activos con los excipientes a temperaturas entre 500 a $1,100{ }^{\circ} \mathrm{C}$. La masa fundida se vierte en moldes para formar un producto final, por ejemplo, varillas de vidrio, tubos de vidrio, discos de vidrio, granulados y bolos monolíticos de liberación controlada. Generalmente se emplean componentes formadores de vidrio de alta solubilidad como óxidos formadores de vidrio (vitrificantes) y óxidos modificadores (fundentes y estabilizantes) que permitan la formación correcta de la red del vidrio ${ }^{(36,37)}$.

\section{Diseño de bolos con minerales traza}

Nuestro grupo de investigación propone un procedimiento simple y económico para elaborar bolos intrarruminales con minerales traza de liberación controlada. Por ejemplo, en el diseño de bolos de selenio, el procedimiento consiste en adicionar cantidades de selenito de sodio, $13 \%$, cutina, $32 \%$; Fe, $54 \%$ y estearato de magnesio, $1 \%$ (Figura 1). Los bolos para corderos pueden tener peso promedio de $8 \mathrm{~g}$, dureza de $21.17 \mathrm{kp}$ y densidad de $2.08 \mathrm{~g}$ $\mathrm{cm}^{-3}$. Los valores recomendados de densidad para su retención en el rumen deben ser

superior a $2.0 \mathrm{~g} \mathrm{~cm}^{-3(8,9,15)}$. El excipiente utilizado para formar la matriz de liberación ha resultado adecuado para liberar Se. 
Figura 1: Diagrama del proceso de elaboración de bolos intrarruminales de selenio por el método de granulación por fusión

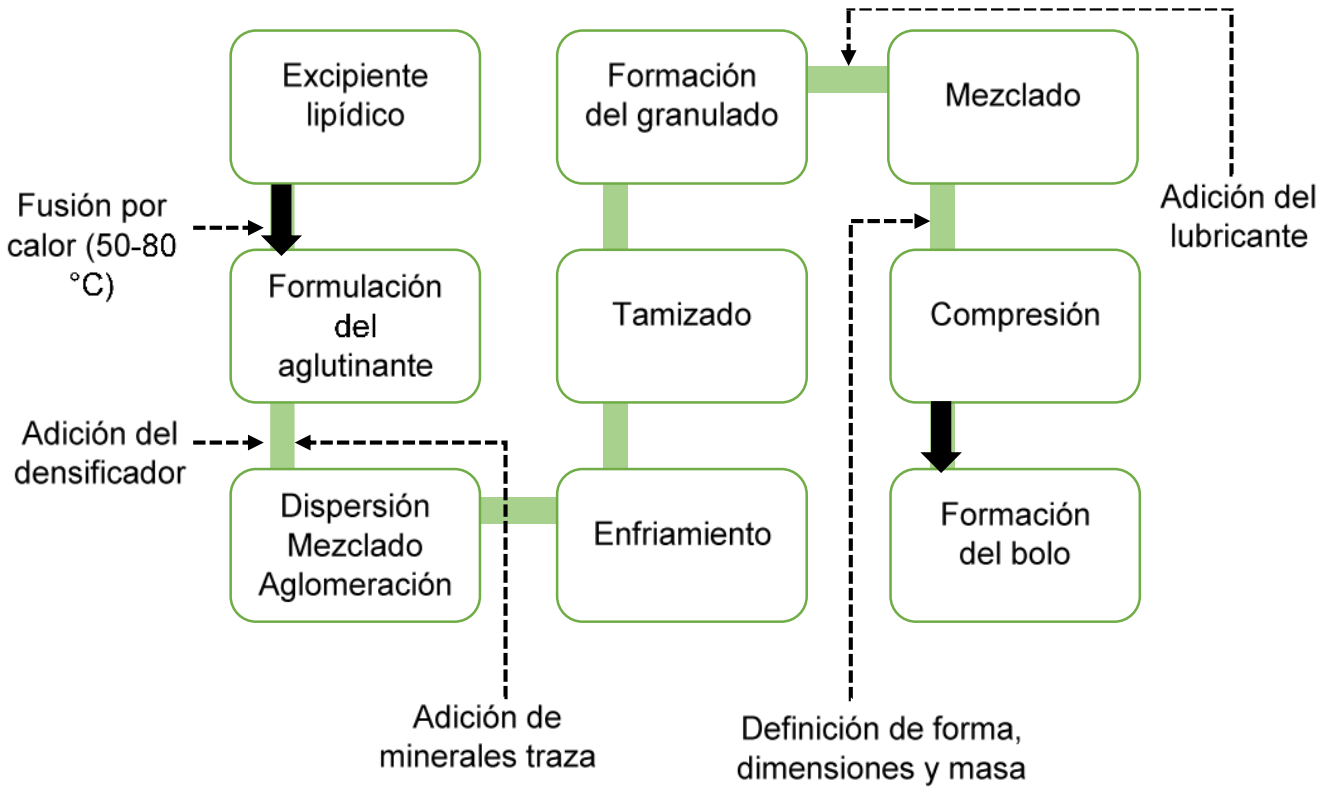

\section{Suplemento de minerales traza con BILC}

$\mathrm{El} \mathrm{Cu}, \mathrm{Zn}, \mathrm{Co}$, Se y I son minerales traza que se han usado para elaborar BILC ${ }^{(6,11,21,24,30)}$. Existen varios tipos de bolos comerciales con diferentes diseños, forma, tamaño, peso, concentraciones variables de minerales traza (Cuadro 1) y tasas de liberación. Esta última característica es indispensable en el diseño de los bolos; a través de ella se conoce la liberación diaria del mineral y la duración del bolo. En el Reino Unido se ha desarrollado un BVS para corregir las deficiencias de $\mathrm{Cu}$, $\mathrm{Co}$ y $\mathrm{Se}$ en ovinos, el bolo fue diseñado para tener una velocidad de liberación en el rumen de $2.53 \mathrm{mg} \mathrm{cm}^{-2}$ día $^{-1}$ y proporcionar $11 \mathrm{mg}$ de $\mathrm{Cu}, 0.5 \mathrm{mg}$ de Co y $0.21 \mathrm{mg}$ de $\mathrm{Se}$, hasta por seis meses ${ }^{(30,31)}$. Los requerimientos de $\mathrm{Cu}$, Co y Se en un ovino que consume $1.5 \mathrm{~kg}$ MS son de $10.5,0.3$ y $0.3 \mathrm{ppm}$, respectivamente. La deficiencia de $\mathrm{Zn}$ en ovinos ha sido tratada con un BVS que contiene $15.2 \%$ de $\mathrm{Zn}$, $0.5 \%$ de Co y $0.15 \%$ de $\mathrm{Se}^{(32,39)}$. El requerimiento diario es de 20 a $33 \mathrm{mg} \mathrm{Zn} \mathrm{kg}{ }^{-1} \mathrm{MS}^{(52)}$. De acuerdo al fabricante del bolo la liberación de $\mathrm{Zn}$ durante 180 días es de aproximadamente $28 \mathrm{mg}_{\text {día }}{ }^{-1(32)}$, considerando el ejemplo anterior, el ovino demandaría un consumo entre 30 y $49 \mathrm{mg}$ de $\mathrm{Zn}$, con un faltante de 2 y $21.5 \mathrm{mg}$, el cual puede cubrirse con el aporte de la dieta. En otro estudio, se reportó una velocidad de disolución de $326 \mathrm{mg}$ día $^{-1}$, equivalente a liberaciones diarias de $49.3 \mathrm{mg}$ de $\mathrm{Zn}, 1.7 \mathrm{mg}$ de Co y $0.5 \mathrm{mg}$ de Se, de modo que el bolo fue capaz de cubrir el requerimiento diario de los ovinos ${ }^{(11)}$. Sin embargo se debe considerar que la tasa de disolución depende de las condiciones en el rumen como el $\mathrm{pH}$, tipo de alimento, sitio de alojamiento del bolo, contracciones ruminales y de los 
efectos de las abrasiones de otros materiales presentes en el retículo-rumen ${ }^{(8,11,13)}$. Otro tipo de bolo diseñado para ovinos presentó una tasa de liberación promedio en el rumen de $103.55 \mathrm{mg} \mathrm{día}^{-1}$, equivalente al suministro diario de $23.01 \mathrm{mg}$ de $\mathrm{Zn}, 0.535 \mathrm{mg}$ de Co y $0.258 \mathrm{mg}$ de $\mathrm{Se}^{(53)}$.

Con la tecnología del vidrio soluble se han diseñado bolos de $100 \mathrm{~g}$ con $13.4 \% \mathrm{de} \mathrm{Cu}$, $0.5 \%$ de Co y $0.30 \%$ de Se para el ganado bovino en pastoreo; de acuerdo con el fabricante, dos bolos liberaron 156, 5.9 y $3.4 \mathrm{mg} \mathrm{día}^{-1} \mathrm{de} \mathrm{Cu}$, Co y Se, respectivamente ${ }^{(33)}$. El requerimiento de un bovino de $500 \mathrm{~kg}$ que consume $10 \mathrm{~kg}$ MS es de 100, $2.5 \mathrm{y} 3 \mathrm{mg}$ día $^{-1}$ de $\mathrm{Cu}$, Co y Se respectivamente ${ }^{(54)}$; por lo tanto, los BVS son efectivos para tratar las deficiencias de minerales traza ${ }^{(55,56,57)}$. La liberación diaria reportada de dos bolos comerciales All-Trace ${ }^{\circledR}$ con minerales traza y vitaminas fue de $138 \mathrm{mg}$ de $\mathrm{Cu}, 113 \mathrm{mg}$ de Zn, $71 \mathrm{mg}$ de Mn, $2.1 \mathrm{mg}$ de I, $2.0 \mathrm{mg}$ de Co, $2.0 \mathrm{mg}$ de Se, 4,644, 929 y 9 UI de vitaminas $\mathrm{A}, \mathrm{D}$ y $\mathrm{E}$ durante un periodo de ocho meses ${ }^{(58)}$; estos bolos fueron diseñados para ser utilizados en ganado bovino con más de $150 \mathrm{~kg}$ de PV. Mientras que la liberación diaria de dos bolos para becerros en crecimiento fue de $60 \mathrm{mg}$ de $\mathrm{Cu}, 1.0 \mathrm{mg}$ de Co, $0.6 \mathrm{mg}$ de $\mathrm{Se}$, $36.8 \mathrm{mg}$ de $\mathrm{Mn}, 53.3 \mathrm{mg}$ de Zn, $1.25 \mathrm{mg}$ de I, 3033 UI de vitamina A, 607 UI de vitamina D y $9.1 \mathrm{UI}$ de vitamina $\mathrm{E}^{(26)}$. Aproximadamente la mitad del peso de la matriz $(30 \mathrm{~g})$ del bolo se libera en las primeras seis semanas y posteriormente la velocidad de liberación disminuye hasta los 7 meses $^{(27)}$.

Cuadro 1: Concentración de minerales traza en diferentes tipos de bolos intrarruminales de liberación controlada

\begin{tabular}{lllllll}
\hline Producto & $\begin{array}{l}\text { Cobre } \\
(\mathbf{m g})\end{array}$ & $\begin{array}{l}\text { Zinc } \\
(\mathbf{m g})\end{array}$ & $\begin{array}{l}\text { Cobalto } \\
(\mathbf{m g})\end{array}$ & $\begin{array}{l}\text { Selenio } \\
(\mathbf{m g})\end{array}$ & $\begin{array}{l}\text { Yodo } \\
(\mathbf{m g})\end{array}$ & $\begin{array}{l}\text { Manganeso } \\
(\mathbf{m g})\end{array}$ \\
\hline Cosecure $^{*(34,38)}$ & $4356-4900$ & $/$ & $165-220.5$ & $49.5-92.75$ & $/$ & $/$ \\
Zincosel $^{(11,32,35,53)}$ & $/$ & $3764-5040$ & $94.1-171.6$ & $43.29-49.5$ & $/$ & $/$ \\
Cosecure $^{* *(33,55)}$ & 13400 & $/$ & 500 & 300 & $/$ & $/$ \\
All-Trace $^{(58)}$ & 16200 & 13320 & 236 & 251 & 497 & 8280 \\
smAll-Trace $^{(26,27)}$ & $5300-5427$ & $4700-4797$ & 90 & $50-54$ & $100-225$ & $3200-3312$ \\
Tracesure $^{(59)}$ & $/$ & 7 & 1000 & 1000 & 6800 & $/$ \\
Ferrobloc $^{(60)}$ & 333 & 36 & 60 & 8 & 24 & 160 \\
BILC $^{+(61)}$ & 3944 & 4366 & 95 & 45 & 330 & 3013 \\
BILC $^{++}(62)$ & $/$ & $/$ & 582 & 148 & 2908 & 11853 \\
\hline
\end{tabular}

*BVS para ovinos; **BVS para bovinos; ${ }^{+}$BILC para ovinos; ${ }^{++}$BILC para bovinos.

Algunos trabajos indican que el suministro de minerales traza mediante BILC aumentan la concentración de minerales en la sangre ${ }^{(30,31,35)}$, mejoran los parámetros productivos y reproductivos $^{(32,35,38,39)}$ y favorecen la respuesta inmune humoral en los rumiantes ${ }^{(11)}$. Estudios han confirmado la efectividad de los BVS en el suministro de $\mathrm{Cu}$, Co y Se para 
corregir y prevenir la deficiencia de estos elementos en ovejas en pastoreo hasta por un año ${ }^{(30,31)}$. Además incrementaron el contenido de Se en el feto y en los corderos recién $\operatorname{nacidos}^{(38)}$.

Los comprimidos con $25 \%$ de $\mathrm{CuO}$, equivalente a $19.3 \%$ de $\mathrm{Cu}$ en la matriz activa, aumentaron las concentraciones de $\mathrm{Cu}$ en sangre de 10.4 a $14.0 \mu \mathrm{mol} \mathrm{L}^{-1}$ y en hígado de 120 a $684 \mathrm{mg} \mathrm{kg}^{-1} \mathrm{MS}$ en ovinos en crecimiento ${ }^{(45)}$. Corderas en semi-estabulación se le administró comprimidos de $5 \mathrm{~g}$ con 1 y $4.6 \%$ de Se, los cuales fueron efectivos hasta por 90 días; sin embargo, se encontró en promedio mayor concentración de Se en sangre (182.01 $\mathrm{ng} \mathrm{g}^{-1}$ ) en las corderas que recibieron el bolo con $4.6 \% \mathrm{de} \mathrm{Se}^{(10)}$. Se ha descrito que los comprimidos fabricados con cemento pega-azulejo con 5 y $10 \%$ de Se, mantienen concentraciones adecuadas de Se en sangre (148.49 y $158.48 \mathrm{ng} \mathrm{g}^{-1}$ ) hasta por 120 días $^{(63)}$. En otro estudio, la predicción de liberación del Se de un bolo con $5.23 \%$ de selenito de sodio fue de $0.177 \mathrm{mg} \mathrm{día}^{-1}$. Los bolos incrementaron el contenido de Se hemático en las ovejas después de 30 días de su aplicación ${ }^{(24)}$. En ovejas Afshari, Abdollahi et al ${ }^{(60)}$ observaron aumentos de $\mathrm{Cu}$, Se y I el día del apareamiento y a los 90 y 100 días de la gestación, usando BILC con múltiples minerales ( $\mathrm{Ca}, \mathrm{Mg}, \mathrm{Na}, \mathrm{Cu}, \mathrm{Mn}, \mathrm{I}, \mathrm{Fe}, \mathrm{Co}, \mathrm{Zn}$ y $\mathrm{Se}$ ). Se ha sugerido que el Se, I, Fe, Zn y Mn son esenciales para la supervivencia del embrión y el desarrollo del feto ${ }^{(64)}$. En general la administración de un BILC con múltiples minerales traza a ovejas gestantes incrementa los contenidos de $\mathrm{Zn}, \mathrm{Cu}$, $\mathrm{Co}$ y $\mathrm{Se}$ en los corderos nacidos ${ }^{(61)}$. El uso de BVS que contienen $\mathrm{Cu}, \mathrm{Zn}$, Co y Se han sido utilizados ampliamente para corregir la deficiencia de minerales traza en corderos semiestabulados ${ }^{(6,32,39)}$, ovejas en pastoreo $^{(34)}$, cabras en condiciones de traspatio ${ }^{(65,66)}$, camellos en crecimiento $^{(55,67)}$ y en bovinos en pastoreo ${ }^{(30,68,69)}$.

\section{Efecto de los BILC sobre los parámetros productivos y reproductivos}

Existen pocos trabajos publicados sobre los efectos de los BILC sobre los parámetros productivos y reproductivos de los rumiantes. En un estudio realizado con becerros Holstein-Friesian destetados y alimentados con una dieta balanceada, recibieron dos bolos con múltiples minerales traza y vitaminas. Los becerros obtuvieron mayor ganancia diaria de peso $(0.59 \mathrm{~kg})$ respecto a los que no recibieron bolo $(0.53 \mathrm{~kg})^{(26)}$. Resultados similares se

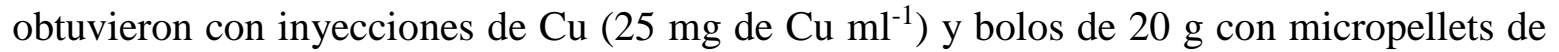
$\mathrm{CuO}$. Los autores no encontraron diferencias en la ganancia diaria de peso (564.2 g día ${ }^{-1}$ ) en becerros de la raza Aberdeen Angus y Hereford, sin embargo sugirieron que los bolos de $\mathrm{CuO}$ son una alternativa para prevenir las deficiencias de $\mathrm{Cu}^{(70)}$. Los corderos que recibieron suplemento de $\mathrm{Zn}$, Co y Se con BVS presentaron mejor ganancia diaria de peso $(153.22 \mathrm{~g})$ respecto al grupo testigo $(136.61 \mathrm{~g})$, con diferencias significativa en la última fase de engorda ${ }^{(11)}$. Por otra parte, se ha reportado que BILC con múltiples minerales han 
mejorado el rendimiento y la calidad nutritiva del calostro $(6.70 \%$ proteína, $6.92 \%$ grasa y $0.59 \%$ materia inorgánica) de las ovejas de la raza Najdi; lo anterior se reportó con mejor salud y crecimiento de los corderos hasta el destete ${ }^{(61)}$. Aliarabi et al $l^{(53)}$ reportaron pesos al nacimiento de $4.63 \mathrm{~kg}$ y ganancia diaria de peso de $0.243 \mathrm{~kg}$ en corderos nacidos de madres tratadas con un bolo que contenía $20 \%$ de Zn, $0.50 \%$ de Co y $0.23 \%$ de Se. El suplemento de minerales traza y vitaminas mediante un BILC aumentó la producción láctea con $8.19 \mathrm{~kg}$ día $^{-1}$ vaca $^{-1(62)}$.

Las ovejas tratadas con un BILC de minerales traza y vitaminas presentaron tasas de nacimientos gemelares de $65.5 \%$, mientras que las ovejas que recibieron una inyección de $\mathrm{Cu}(12.5 \mathrm{mg} \mathrm{Cu})$ presentaron $44.8 \%$ de nacimientos gemelares. En busca de confirmar el efecto de los bolos, los autores dosificaron un BILC a ovejas con deficiencia de $\mathrm{Cu}$ y $\mathrm{Se}$, mejorando los nacimientos gemelares en $59 \%$, mientras que el grupo sin dosificación presentó $11 \%$ de ovejas estériles ${ }^{(27)}$. En otro estudio obtuvieron $80 \%$ de nacimientos múltiples por efecto de la administración de dos BILC a ovejas previo a la sincronización $^{(60)}$. El ganado lechero en pastoreo suplementado con dos BVS que contenía $13.4 \%$ de $\mathrm{Cu}$, presentó disminución en el número de servicio a la concepción $(2.5 \pm 0.3$ a $1.7 \pm 0.2)$ y el intervalo de parto se redujo de 407 a 371 días $^{(68)}$.

El suplemento de Zn, Co y Se a través de BVS han mejorado la motilidad y la proporción de espermatozoides vivos, así como la integridad de las membranas espermáticas ${ }^{(35,39)}$. El Zn está involucrado en el catabolismo de los lípidos localizados en la parte intermedia del esperma, para generar la energía que se necesita para la movilidad de los espermatozoides $^{(71)}$. El $\mathrm{Cu}, \mathrm{Zn}$ y Se tienen propiedades antioxidantes y pueden actuar para reducir las especies de oxígeno reactivo y evitar el daño a los espermatozoides ${ }^{(72,73)}$. En otra investigación se evaluó el efecto de un bolo con 500 mg de Se sobre la calidad del semen de ovinos Hampshire y Suffolk; los bolos aumentaron la actividad de GSH-Px, la movilidad, el número de espermatozoides vivos y la viabilidad del eyaculado de los sementales ${ }^{(74)}$.

\section{Efecto de los BILC sobre la sanidad animal}

La deficiencia de Se es un problema grave que afecta la producción animal. Especialmente en los ovinos la tasa de mortalidad de corderos durante la etapa perinatal y neonatal es de $62 \%{ }^{(75)}$, principalmente son evidentes los signos de la enfermedad del músculo blanco o distrofia muscular nutricional ${ }^{(4)}$. En un estudio con ovejas suplementadas con un BVS antes del parto, se logró disminuir la incidencia de signos de la enfermedad del músculo blanco en los corderos nacidos ${ }^{(31)}$. El bolo liberó $0.21 \mathrm{mg}$ de Se día ${ }^{-1}$, cantidad suficiente para ser transferido a la placenta o al calostro que recibieron los corderos en las primeras horas de $\operatorname{vida}^{(31,56)}$. La concentración de Se en la leche proveniente de vacas suplementadas con un 
BVS fue mayor $\left(0.0658 \mu \mathrm{g} \mathrm{g}^{-1}\right)$ respecto al grupo testigo $\left(0.0374 \mu \mathrm{g} \mathrm{g}^{-1}\right)$ durante siete meses $^{(56)}$. Otro estudio con corderos nacidos de ovejas tratadas con un BVS que contenía $20 \%$ de $\mathrm{Zn}, 0.50 \%$ de Co y $0.23 \%$ de Se, no presentaron incidencia de la enfermedad del músculo blanco, mientras que los corderos nacidos de las madres no tratadas alcanzaron una tasa de mortalidad de $10.3 \%$ y signos de la enfermedad del músculo blanco por $18 \%$.

Con respecto al papel de los minerales traza en la función inmune, se ha demostrado que los bolos de $\mathrm{Zn}$ provocan efecto positivo en los corderos sobre su respuesta inmune humoral ante el antígeno hemocianina de lapa californiana -KLH. Sin embargo se cree que la respuesta inmune fue favorecida por acción conjunta del $\mathrm{Zn}$, Co y Se presentes en el bolo $^{(11)}$. En camellos, se encontró efecto similar en la respuesta inmune humoral a la inyección intravenosa de $2 \mathrm{~mL}$ de suspensión de glóbulos rojos de ovejas al $20 \%$. Además, se informó que el BVS mejoró la inmunidad mediada por células, incrementando el grosor de los pliegues cutáneos después de $24 \mathrm{~h}$ de la inyección de fitohemaglutinina (PHA) ${ }^{(67)}$. En cabritos se ha demostrado que el tratamiento con un bolo de selenito de sodio y una inyección de $\mathrm{Se}\left(0.25 \mathrm{mg} \mathrm{kg}{ }^{-1} \mathrm{PV}\right)$ mejoraron la respuesta inmune humoral a la inmunización con una bacteria-toxoide (Toxo Bac Neumonias) ${ }^{(76)}$.

Munday et $a l^{(28)}$ desarrollaron un bolo de $\mathrm{Zn}$ para proteger a los corderos contra el eccema facial. El bolo consiste en un núcleo de $43 \mathrm{~g}$ de $\mathrm{ZnO}$ cubierto por un revestimiento impermeable al agua, excepto un extremo que al entrar en contacto con el líquido ruminal se erosiona para liberar una dosis de $\mathrm{Zn}$ de $20 \mathrm{mg} \mathrm{kg}^{-1}$ día ${ }^{-1}$. Las cantidades de inclusión de 54, 81 y $108 \mathrm{~g} \mathrm{Zn}$ en los bolos para ovejas presentaron beneficio protector contra la esporidesmina producida por el hongo Pithomyces chartarum $^{(77)}$. La administración de bolos de $\mathrm{ZnO}$ a becerros expuestos a la esporidesmina, presentaron menor actividad sérica de la gama-glutamiltransferasa respecto al grupo testigo; esto demostró la efectividad de los bolos para reducir la incidencia y la gravedad del eccema facial ${ }^{(29)}$.

\section{Conclusiones}

Las innovaciones tecnológicas que se utilizan en los procesos de elaboración de productos farmacéuticos de uso veterinario pueden contribuir a mejorar los métodos de suplementación mineral. Los BILC son el método más eficaz para proporcionar minerales traza hasta por más de un año. Los mecanismos de liberación controlada que presentan los bolos intrarruminales son prácticos para corregir y prevenir las deficiencias de minerales traza en los rumiantes en pastoreo. Hay evidencias positivas donde los BILC mejoran los parámetros productivos y reproductivos, así como la actividad inmune humoral de los rumiantes. El uso de BILC disminuye el manejo de los animales, el estrés y la disminución de los costos económicos. Sin embargo, en América Latina no es un método común para 
suministrar los minerales traza, por ello es necesario más investigación en el desarrollo de los BILC de acuerdo con el perfil deficiente de los suelos y forrajes dedicados al pastoreo.

\section{Literatura citada:}

1. McDowell LR, Arthington JD. Minerales para rumiantes en pastoreo en regiones tropicales. 4ta ed. Universidad de Florida. Gainsville, Florida. USA; 2005.

2. Ramírez-Orduña R. Ramírez RG, González RH, Haenlein GFW. Mineral content of browse species from Baja California Sur, México. Small Ruminant Res 2005;(57):110.

3. Gámez BJR. Diagnóstico del estado mineral de bovinos en San Juan Del Río, Choapam, Oaxaca [tesis maestría]. Chapingo, México: Universidad Autónoma Chapingo; 2009.

4. Ramírez-Bribiesca JE, Tórtora JL, Huerta M, Aguirre A, Hernández LM. Diagnosis of selenium status in grazing dairy goats on the Mexican plateau. Small Ruminant Res 2001;(41):81-85.

5. Vieyra-Alberto R, Domínguez-Vera IA, Olmos-Oropeza G, Martínez-Montoya JF, Borquez-Gastelum JL, Palacio-Núñez J, et al. Perfil e interrelación mineral en agua, forraje y suero sanguíneo de bovinos durante dos épocas en la huasteca potosina, México. Agrociencia 2013;(47):121-133.

6. Kendall NR, Mackenzie AM, Telfer SB. Effect of a copper, cobalt and selenium soluble glass bolus given to grazing sheep. Livest Prod Sci 2001;(68):31-39.

7. Grace DN, Knowles SO. Trace supplementation of livestock in New Zealand: meeting the challenges of free-range grazing systems. Vet Med Int 2012;(ID 639472):8.

8. Cardinal JR. Intraruminal decives. In: Rathbone MJ editor. Adv Drug Deliv Rev 1997;(28):303-322.

9. Rathbone MJ, Cardinal JR, Ogle CR. Mechanisms of drug release from veterinary drug delivery systems. In: Rathbone MJ, Gurny R editors. Controlled release veterinary delivery. Biol Pharm Consid: Elsevier Sci; 2000:17-50.

10. Blanco OMA, Spross SAK, Rosiles MR. Evaluación de comprimidos intrarruminales de selenio por concentración sanguínea y lanar de corderas semiestabuladas. Vet Méx 2000;31(2):121-127.

11. Kendall NR, Mackenzie AM, Telfer SB. The trace element and humoral immune response of lambs administered a zinc, cobalt and selenium soluble glass bolus. Livest Sci 2012;(148):81-86. 
12. Fan LT, Singh SK. Contolled release: A quantitative treatment. Polymers properties and applications. Berlin Heidelberg: Springer-Verlag; 1997.

13. Cardinal JR. Intraruminal controlled release boluses. In: Rathbone MJ, Gurny R editors. Controlled release veterinary drug delivery. Biol Pharm Con; Elsevier Sci 2000;(36):51-82.

14. Vandamme TF, Rathbone MJ. Long acting rumen drug delivery systems. In: Rathbone MJ, McDowell A editors. Long acting animal health drug products: Fundamentals and applications. Adv Deliv Sci Tech; Springer 2013:221-246.

15. Riner RL, Byford LG, Stratton JA, Hair JA. Influence of density and location on degradation of sustained release boluses given to cattle. Am J Vet Res 1982;43(11):2028-2030.

16. Marston HR. Therapeutic pellets for ruminants. U.S. Patent 3056 724, 1962.

17. Andrews ED, Isaacs CE, Findlay RJ. Response of cobalt deficient lambs to cobaltic oxide pellets. NZ Vet J 1958;6(5):140-146.

18. Langlands JP, Bowles JE, Donald GE, Smith AJ. Selenium supplements for grazing sheep. 2. Effectiveness of intra-ruminal pellets. Anim Feed Sci Tech 1990;(28):15-28.

19. Millar KR, Meads WJ. The efficacy of intraruminal pellets composed of elemental selenium and iron in sheep. NZ Vet J 1988;36(2):53-55.

20. Langlands JP, Donald GE, Bowles JE, Smith AJ. Selenium supplements for grazing sheep. 1. A comparison between soluble salts and other forms of supplement. Anim Feed Sci Tech 1990;(28):1-13.

21. Judson GJ. Trace element supplements for sheep at pasture. In: Masters DG, White CL editors. Detection and treatment of mineral nutrition problems in grazing sheep. Australian Centre for International Agricultural Research, Canberra, Australia: ACIAR Monograph 1996;(37):57-80.

22. Díaz SVM. Efectos de bolos intrarruminales de sulfas y selenio para el control de la coccidiosis caprina [tesis licenciatura]. Cuautitlán Izcalli, Edo. de México: Universidad Nacional Autónoma de México; 2012.

23. Evrard B, Delahaut P, Hubert P, Crommen J, Delattre L. Biopharmaceutical aspects of the development af a sulfamethazine oral sustained release bolus for lambs. J Contr Rel 1995;(35):107-115. 
24. Revilla-Vázquez A, Ramírez-Bribiesca E, López-Arellano R, Hernández-Calva LM, Tórtora-Pérez J, García-García E, et al. Suplemento de selenio con bolos intrarruminales de selenito de sodio en ovinos. Agrociencia 2008;(42):629-635.

25. Hemingway RG, Ritchie NS, Parkins JJ, Device for introducing nutrients and/or therapeutic materials into ruminant animals. U.S. Patent 4 732 764, 1988.

26. Hemingway RG, Parkins JJ, Ritchie NS. Sustained-release boluses to supply trace elemnts and vitamins to calves. Vet J 1997;(153):221-224.

27. Hemingway EG, Parkins JJ, Ritchie NS. Enhanced reproductive performance of ewes given a sustained-release multi-trace element/vitamin ruminal bolus. Small Ruminant Res 2001;(39):25-30.

28. Munday R, Thompson AM, Fowke EA, Wesselink C, Smith BL, Towers NR, et al. Azinc-containing intraruminal device for facial eczema control in lambs. NZ Vet J 1997;45(3):93-98.

29. Munday R, Thompson AM, Smith BL, Towers NR, O'Donnell K, McDonald RM, et al. Azinc-containing intraruminal device for prevention of the sporidesmin-induced cholangiopathy of facial eczema in calves. NZ Vet J 2001;49(1):29-33.

30. Telfer SB, Zervas G, Carlos G. Curing or preventing deficiencies in dopper, cobalt and selenium in cattle and sheep using tracerglass. Can J Anim Sci 1984;64(Suppl):234235.

31. Zervas G. Treatment of dairy sheep with soluble glass boluses containing copper, cobalt and selenium. Anim Feed Sci Technol 1988;(19):79-83.

32. Kendall NR, Telfer SB. Induction of zinc deficiency in sheep and its correction with a soluble glass bolus containing zinc. Vet Rec 2000;(146):634-637.

33. Sprinkle JE, Cuneo SR, Frederick HM, Enns RM, Schafer DW, Carstens GE, et al. Effects of long acting trace mineral rumen bolus upon range cow productivity. Proc Western Section, Am Soc Anim Sci 2004;(54).

34. Kendall NR, Jackson DW, Mackenzie AM, Illingworth DV, Gill IM, Telfer SB. The effect of a zinc, cobalt and selenium soluble glass bolus on the trace element status of extensively grazed sheep over winter. Anim Sci 2001;(73):163-169.

35. Kendall NR, McMullen S, Green A, Rodway RG. The effect of zinc, cobalt and selenium soluble glass bolus on trace element status and semen quality of ram lambs. Anim Reprod Sci 2000;(62):277-283. 
36. Telfer SB, Zervas G, Knott P. Water soluble glass articles, their manufacture, and their use in the trearment of ruminant animals. U. S. Patent 4482 541, 1984.

37. Drake CF. Controlled release glass. U.S. Patent 4350 675, 1982.

38. Zervas G, Telfer SB, Carlos G, Anderson P. The effect of soluble-glass boluses containing copper, cobalt and selenium on the blood composition of ewes. Anim Feed Sci Technol 1988;(21):23-29.

39. Kendall NR, Green A, McMullen S, Rodway RG. The effect of a zinc, cobalt, and selenium bolus on ram semen quality and trace element status. In: Roussel AM, et al, editors. Trace elements in man and animals. Boston, MA. Springer, 2002.

40. Sakkinen H, Eloranta E, Vahtila S, Puukka M, Timisjarvi J, Saarela S, et al. Effects of magnesium oxide and magnesium alloy rumen boluses on plasma and urinary magnesium and calcium concentrations in reindeer (Rangifer tarandus tarandus). Small Riminant Res 2004;(54):69-79.

41. Vandamme TF, Ellis KJ. Issues and challenges in developing ruminal drug delivery systems. Adv Drug Deliv Rev 2004;(56):1415-1436.

42. Dewey DW. An effective method for the administration of trace amounts of copper to ruminants. Search 1977;(8):326-327.

43. Langlands JP, Donald GE, Bowles JE, Smith AJ. Trace element nutrition of grazing ruminants. 3. Copper oxide powder as a copper supplement. Austr J Agr Res 1989;(40):187-193.

44. Burke JM, Miller JE. Control of Haemonchus contortus in goats with a sustainedrelease multi-trace element/vitamin ruminal bolus containing copper. Vet Parasitology 2006;(142):132-137.

45. Parkins JJ, Hemingway RG, Lawson DC, Ritchie NS. The effectiveness of copper oxide powder as a component of a sustained-release multi-trace element and vitamin rumen bolus system for cattle. Br Vet J 1994;(150):547-553.

46. Huerta BM, Amándola MR, Martínez HPA, García MJG, Sánchez RC, Domínguez VI, et al. Suplementos minerales para rumiantes. Postgrado de Producción Animal, Departamento de Zootecnia. Universidad Autónoma de Chapingo; 2010.

47. Patil H, Tiwari RV, Repka MA. Hot-melt extrusion: from theory to application in pharmaceutical formulation. AAPS Pharm Sci Tech 2016;(17):20-42. 
48. Repka MA, Bandari S, Raman KV, Vo AQ, McFall H, Pimparade MB, et al. Melt extrusión with poorly soluble drugs-an integrated review. Int J Pharm 2018;(535):6885.

49. Crowley MM, Zhang F, Repka MA, Thumma S, Upadhye SB, Kumar BS, et al. Pharmaceutical applications of hot-melt extrusión: Part I. Drug develop and Pharm 2007;(33):909-926.

50. Ochoa DL, Igartua OM, Hernández MRM, Gascón RA, Pedraz MJL. Granulación por fusión en mezcladores granuladores de alta velocidad. VITAE, Rev Fac Quím Farm 2006;(13):40-47.

51. Rodríguez GA. Validación del proceso de fabricación de bolos intrarruminales de selenio inorgánico. [tesis licenciatura] Cuautitlán Izcalli, Edo. de México: Facultad de Estudios Superiores Cuautitlán, Universidad Nacional Autónoma de México; 2006.

52. NRC. National Research Council. Nutrient requirements of small ruminants: sheep, goats, cervids and new world camelids. Washington, DC, USA: National Academy Press; 2007.

53. Aliarabi H, Fadayifar A, Alimohamady R, Hossein DA. The effect of maternal supplementation of zinc, selenium, and cobalt as slow-release ruminal bolus in late pregnancy on some blood metabolites and performance of ewes and their lambs. Biol Trace Elem Res 2019;(187):403-410.

54. NRC. National Research Council. Nutrient Requirements of Beef Cattle. 7th rev. ed. Washington, DC, USA: National Academy Press, 2000.

55. Liu ZP, Xiong GL. The effect of a copper, selenium and cobalt soluble glass bolus on the trace element status of Bactrian camels. J Anim Feed Sci 2007;(Suppl 1):313-317.

56. Hidiroglou M, Proulx J, Jolette J. Effect of intraruminally administered, selenium soluble-glass boluse on selenium status in cows and their calves. J Anim Sci 1987;(65):815-820.

57. Hidiroglou M, Proulx J. Evaluation of a long-acting selenium and copper preparation for intraruminal adminitration to cattle. Ann Rech Vet 1988;(19):187-191.

58. Baumgurtel KL, Judson GJ. Evaluation of a sustained release bolus to supply trace elements and vitamins beef cattle. Anim Prod Austr 1998;(22):133-136.

59. Rose M, Pearson S, Cratchley T. Effect of iodine, selenium and cobalt rumen boluses given to dry dairy cows on the immunoglobulin and thyroid hormone status of calves. Anim Sci J 2012;(83):543-548. 
60. Abdollahi E, Kohram H, Shahir MH, Nemati MH. The influence of a slow-release multi-trace element ruminal bolus on trace element status, number of ovarian follicles and pregnancy outcomes in synchronized Afshari ewes. Iranian $\mathbf{J}$ Vet Res 2015;16(1):63-68.

61. Abdelrahman MM, Aljumaah RS, Ullah KR. Effects of prepartum sustained-release trace elements ruminal bolus on performance, calustrum composition and blood metabolites in Najdi ewes. Environ Sci Pollut Res 2017;(24):9675-9680.

62. Pulgar AR, Vera VR, Serrano CE. Efectos de la utilización de bolos intraruminales a base de oligoelementos, sobre indicadores reproductivos y productivos en vacas lecheras de alta producción. Sitio Arg Prod Anim 2013;51-52.

63. Gutiérrez OC, Spross SAK, Rosiles MR, Ducoing WA, Ortiz HA. Selenio sanguíneo y fecal en ovinos a partir de comprimidos inorgánicos intrarruminales. Vet Mex 2005;36(3):313-324.

64. Hostetler CE, Kincaid RL, Mirando MA. The role of essential trace elements in embryonic and fetal development in livestock. Vet J 2003;(166):125-139.

65. Serra AB, Serra SD, Nakamura K, Orden EA, Cruz LC, Fujihara T. Effect of selenium in soluble glass bolus on selenium content of milk and blood of goats. Biol Trace Elem Res 1996;(55):207-212.

66. Hayashida M, Orden EA, Cruz EM, Cruz LC, Fujihara T. Effects of intraruminal soluble glass bolus on blood selenium and plasma mineral level of grazing does under Backyard conditions in selected áreas in Nueva Ecija, Philippines. Asian-Aust J Anim Sci 2003;16(2):187-197.

67. Alhidary IA, Abdelrahman MM, Harron RM. Effects of a long-acting trace mineral rumen bolus supplement on growth performance, metabolic profiles, and trace mineral status of growing camels. Trop Anim Health Prod 2016;(48):763-768.

68. Moeini MM, Telfer SB, Sanjabi MR. The effect of Cosecure ${ }^{\circledR}$ supplementation on the copper status and fertility of grazing Holstein-Friesian dairy cattle. Acta Vet Scand 2003;(Suppl 98):257.

69. Sprinkle JE, Cuneo SP, Frederick HM, Enns RM, Schefer DW, Carstens GE, et al. Effect of a long-acting, trace mineral, reticulorumen bolus a range cow productivity and trace mineral profiles. J Anim Sci 2006;(84):1439-1453.

70. Pechin GH, Sánchez LO, Cseh S. Evaluación de dos formas de administración (bolos de liberación lenta vs. EDTA $\mathrm{Cu}$ inyectable) en la prevención de la deficiencia de cobre en bovinos para carne. Ciencia Vet 2006;8(1):5-15. 
71. Roy B, Baghel RPS, Mohanty TK, Mondal G. Zinc and male reproduction in domestic animals: A review. Indian J Anim Nutr 2013;(30):339-350.

72. Suttle NF. Mineral nutrition of livestock. 4th ed. London, UK. CABI Publishing. United Kingdom; 2010.

73. Zhao CY, Tan SX, Xiao XY, Qiu XS, Pan JQ, Tang ZX. Effects of dietary zinc oxide nanoparticles on growth performance and antioxidative status in broilers. Biol Trace Elem Res 2014;(160): 361-377.

74. Carrillo-Nieto O, Domínguez-Vera IA, Huerta-Bravo M, Jaramillo-Escutia G, DíazZarco S, Vázquez-Armijo JF, et al. Actividad de GSX-Px, concentración de selenio y calidad del eyaculado en sementales ovinos suplementados con selenio durante la época reproductiva. Agrociencia 2018;(52):827-839.

75. Ramírez-Bribiesca E, Hernández-Camacho E, Hernández-Calva LM, Tórtora-Pérez JL. Efecto de un suplemento parenteral con selenito de sodio en la mortalidad de corderos y los valores hemáticos de selenio. Agrociencia 2004;(38):43-51.

76. Díaz-Sánchez V, Rodríguez PG, Ramírez-Bribiesca E, Morales-Álvarez J, LópezArrellano R. Evaluación de bolos selenio sobre parámetros productivos e IgG en cabritos inmunizados con bacterina-toxoide. Abanico Veterinario 2018;8(3):118-129.

77. Bennison JJ, Nottingham RM, Key EL, Parkins JJ. The effect of zinc oxide and elemental zinc boluses on the concentrations of $\mathrm{Zn}$ in serum and faeces, and on providing protection from natural Pithomyces chartarum challenge in sheep. NZ Vet J 2010;58(4):201-206. 